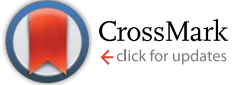

Cite this: J. Mater. Chem. A, 2017, 5, 2739

Received 1st May 2016

Accepted 26th December 2016

DOI: $10.1039 / \mathrm{c} 6 \mathrm{ta} 03631 \mathrm{~g}$

www.rsc.org/MaterialsA

\title{
Tuning of graphene oxide composition by multiple oxidations for carbon dioxide storage and capture of toxic metals $\dagger$
}

\author{
Michal Nováček, ${ }^{a}$ Ondřej Jankovský, ${ }^{a}$ Jan Luxa, ${ }^{a}$ David Sedmidubský, ${ }^{a}$ \\ Martin Pumera, ${ }^{b}$ Vlastimil Fila, ${ }^{c}$ Miloslav Lhotka, ${ }^{c}$ Kateřina Klímová, ${ }^{a}$ \\ Stanislava Matějkovád and Zdeněk Sofer ${ }^{\star a}$
}

Graphene oxide $(\mathrm{GO})$ is a material used as a precursor for the synthesis of graphene and its derivatives. Chemical properties of graphene are strongly influenced by the chemical composition of the original GO. In this paper we would like to show that the amount as well as the type of functional groups can be significantly increased and controlled by multiple oxidations of GO. For this purpose we performed multiple oxidations using two chlorate methods (Staudenmaier and Hofmann) and a permanganate method (Hummers). The results show a possibility of tuning the composition of GO functionalities by multiple oxidations. The obtained results also show that the second and third subsequent reoxidation reactions significantly increase the amount of oxygen containing groups in GO, mainly carboxylic groups. The multiple oxidation of graphene oxide led to a significant increase of carbon storage capacity. The high concentration of oxygen functionalities led to an increase of sorption capacity by more than one order of magnitude.

\section{Introduction}

Graphene has become one of the most intensively studied materials since its isolation in 2004. ${ }^{1}$ It was found that graphene exhibits unique chemical, mechanical, physical, and electronic properties. ${ }^{1}$ Thanks to the ballistic transport of electrons and high electrical conductivity, graphene can be used for the fabrication of transparent electrodes, sensors, solar cells, display devices, energy and halogen storage and various composite materials. ${ }^{2-9}$ Graphene-based materials have also been used for gas sorption, storage and separation. ${ }^{\mathbf{1 0}}$ Also the synthesis of various graphene modifications with unique properties has been reported. ${ }^{\mathbf{1 1 , 1 2}}$

Various methods of graphene synthesis have been reported. One of the possible methods is based on a CVD deposition of carbon on metallic substrates. CVD techniques yield high quality graphene for microelectronic devices. ${ }^{\mathbf{1 3}}$ On the other hand top-down methods are suitable for graphene mass

\footnotetext{
a Department of Inorganic Chemistry, University of Chemistry and Technology Prague, Technická 5, 16628 Prague 6, Czech Republic.E-mail: zdenek.sofer@vscht.cz

${ }^{b}$ Division of Chemistry \& Biological Chemistry, School of Physical and Mathematical Sciences, Nanyang Technological University, Singapore 637371

${ }^{c}$ Department of Inorganic Technology, University of Chemistry and Technology Prague, Technická 5, 16628 Prague 6, Czech Republic

${ }^{d}$ Institute of Organic Chemistry and Biochemistry AS CR, v.v.i., Flemingovo nam. 2., 16610 Prague 6, Czech Republic

$\dagger$ Electronic supplementary information (ESI) available. See DOI: 10.1039/c6ta03631g
}

production. Top down methods can be divided into two different branches: the mechanical exfoliation of graphite and the two step method of oxidation of graphite followed by reduction of the formed graphene oxide. ${ }^{1}$

Graphite oxide can be prepared by the treatment of graphite in an environment of concentrated acids and strong oxidizing agents such as potassium chlorate or potassium permanganate. During this oxidation, functional groups are attached in between graphitic planes, which leads to an increase in interlayer spacing along the $c$ axis. ${ }^{14}$ There are four basic methods used for the synthesis of this material. Two of them, Hofmann's and Staudenmaier's methods use potassium chlorate while Hummers' and modified Hummers' methods use potassium permanganate as an oxidizing agent..$^{15-17}$ In the $19^{\text {th }}$ century, graphite oxide was synthesized from graphite by oxidation using potassium chlorate in fuming nitric acid. ${ }^{18}$ Since that time, some improvements have been made in order to obtain more oxidized graphite oxide: Hofmann and Staudenmaier used a mixture of potassium chlorate, sulfuric acid and nitric acid. ${ }^{15,17-19}$ The prepared material was termed "graphite acid" due to the content of various oxygen containing functionalities including the carboxylic group. ${ }^{17,19}$ Many years later, Hummers developed a novel method where graphite was oxidized by permanganate in sulfuric acid. ${ }^{\mathbf{1 6}} \mathrm{A}$ few years ago, modification of the permanganate based method was reported and the mechanism of graphite oxidation was investigated. ${ }^{20}$

Since graphene oxide is a complex material showing the presence of various oxygen functionalities, complex analytical 
methods are used for its characterization. The analytical methods used typically for organic compounds like NMR are not broadly applied for graphene based materials due to the necessity of measurement in the solid state and relatively low sensitivity. The most broadly used methods are based on FT-IR and X-ray photoelectron spectroscopy. These methods reveal the presence of individual oxygen functionalities and are able to give information about their concentration. ${ }^{17}$ However, the exact information about the overall composition, especially hydrogen concentration, can be most simply obtained by elemental combustion analysis. ${ }^{18}$

Graphene oxide is an important starting material for the synthesis of various graphene derivatives. ${ }^{21}$ Besides this, graphene oxide has a huge application potential in various fields covering energy related applications like electrocatalysis and water splitting. ${ }^{22}$ Other potential applications are focused on the chemical and physical storage of hydrogen, where promising hydrogen storage capacities have been reported. ${ }^{22}$ The presence of oxygen functionalities also provides an opportunity for environmentally related applications of graphene oxide, like wastewater treatment and water purification to remove inorganic as well as various organic contaminants including hormones or antibiotics. ${ }^{23}$ Graphene oxide forms stable suspensions in various polar solvents which can be used for the preparation of transparent electrodes. ${ }^{24}$ Graphene oxide is also broadly used for the synthesis of 3D structures with environmental and energy related applications. ${ }^{25}$

In this contribution, we wish to demonstrate that the chemical composition of graphene oxide can be tuned by multiple subsequent oxidations of graphene oxide. Three singly, doubly and triply reoxidized graphene oxides were synthesized according to Staudenmaier, Hofmann and Hummers methods and characterized in detail by a number of analytical techniques. We demonstrate the application of such multiply oxidized graphene oxide for the sorption of heavy metals and for $\mathrm{CO}_{2}$ and $\mathrm{CH}_{4}$ gas sorption. The high difference in $\mathrm{CO}_{2}$ and $\mathrm{CH}_{4}$ sorption capacity shows promising applications for $\mathrm{CO}_{2}$ storage as well as gas separation. In particular, multiple oxidations led to more than a quintuple increase in the sorption capacity of heavy metals like cadmium. Such results give these materials huge potential in energy as well as environmental related applications.

\section{Experimental}

\section{Synthesis procedures}

We prepared nine different graphite oxides from pure graphite microparticles (2-15 $\mu \mathrm{m}, 99.9995 \%$, from Alfa Aesar). Sulfuric acid (98\%), nitric acid (68\%), fuming nitric acid (>98\%), potassium chlorate (99\%), potassium permanganate $(99.5 \%)$, sodium nitrate $(99.5 \%)$, sodium hydroxide, hydrogen peroxide (30\%), hydrochloric acid (37\%), silver nitrate (99.5\%), barium nitrate $(99.5 \%)$ and $N, N$-dimethylformamide (DMF) were obtained from Penta, Czech Republic.

Graphene oxide prepared by the Staudenmaier method ${ }^{\mathbf{1 3}}$ is termed ST-GO. Sulfuric acid $(98 \%, 87.5 \mathrm{~mL})$ and fuming nitric acid $(>98 \%, 27 \mathrm{~mL})$ were added to a reaction flask containing a magnetic stir bar. Subsequently, the mixture was cooled to $0{ }^{\circ} \mathrm{C}$ and graphite $(5 \mathrm{~g})$ was added. The mixture was vigorously stirred to avoid agglomeration and to obtain a homogeneous dispersion. While keeping the reaction flask at $0{ }^{\circ} \mathrm{C}$, potassium chlorate ( $55 \mathrm{~g}$ ) was slowly added. Upon the complete dissolution of potassium chlorate, the reaction flask was loosely capped to allow the escape of the gas evolved. Then the mixture was continuously stirred for $96 \mathrm{~h}$ at room temperature. The mixture was then poured into deionized water ( $3 \mathrm{~L}$ ) and decanted. Graphene oxide was redispersed in $\mathrm{HCl}$ solution $(5 \%, 3 \mathrm{~L})$ to remove sulfate ions and repeatedly centrifuged and redispersed in deionized water until a negative reaction to chloride and sulfate ions was achieved. Graphene oxide slurry was then dried in a vacuum oven. In the next step the graphene oxide was reoxidized according to the same procedure (graphene oxide STGO from the first oxidation was used instead of graphite). The resulting sample is denoted as ST-GO-2x. Finally, the same procedure was performed for the third time with ST-GO- $2 x$ as the starting material. This sample is labeled ST-GO-3x.

Graphene oxide prepared according to the Hofmann method was termed HO-GO. ${ }^{15}$ Sulfuric acid $(98 \%, 87.5 \mathrm{~mL})$ and nitric acid $(68 \%, 27 \mathrm{~mL})$ were added to a reaction flask (Pyrex beaker with a thermometer) containing a magnetic stir bar. The mixture was then cooled by immersion in an ice bath for 30 min. Graphite (5 g) was then added to the mixture with a vigorous stirring motion. While keeping the reaction flask in the ice bath, potassium chlorate ( $55 \mathrm{~g}$ ) was slowly added to the mixture. Upon the complete dissolution of potassium chlorate, the reaction flask was then loosely capped to allow the escape of the gas evolved and the mixture was continuously stirred for 96 hours at room temperature. The mixture was poured into $3 \mathrm{~L}$ of deionized water and decanted. Graphene oxide was then redispersed in $\mathrm{HCl}$ solution (5\%, $3 \mathrm{~L}$ ) to remove sulfate ions and repeatedly centrifuged and redispersed in deionized water until a negative reaction to chloride and sulfate ions was achieved. Graphene oxide slurry was then dried in a vacuum oven. In the next step the graphene oxide was reoxidized according to the same procedure (except that instead of graphite we used our graphene oxide HO-GO). The sample is termed HO-GO-2x. Similarly, after the third oxidation we prepared the graphene oxide referred to as HO-GO- $3 x$.

The third graphene oxide was synthesized similarly to the Hummers method and is named HU-GO. ${ }^{14}$ Graphite $(5 \mathrm{~g})$ and sodium nitrate $(2.5 \mathrm{~g})$ were stirred with sulfuric acid $(98 \%$, $115 \mathrm{~mL}$ ). The mixture was then cooled to $0{ }^{\circ} \mathrm{C}$. Potassium permanganate $(15 \mathrm{~g}$ ) was then added over a period of two hours. During the next four hours, the reaction mixture was allowed to reach room temperature before being heated to $35{ }^{\circ} \mathrm{C}$ for 30 min. The reaction mixture was then poured into a flask containing deionized water $(250 \mathrm{~mL})$ and heated to $70{ }^{\circ} \mathrm{C}$ for 15 minutes. The mixture was then poured into deionized water $(1 \mathrm{~L})$. The unreacted potassium permanganate and manganese dioxide were removed by the addition of $3 \%$ hydrogen peroxide. The reaction mixture was then allowed to settle and decant. The obtained graphene oxide was then purified by repeated centrifugation and redispersing in deionized water until a negative reaction to sulfate ions was achieved. Graphene oxide slurry was 
then dried in a vacuum oven. In the next step the graphene oxide was reoxidized according to the same procedure (except that instead of graphite we used our graphene oxide HU-GO). This sample is termed HU-GO- $2 x$. Finally, the same procedure was performed for the third time with HU-GO- $2 x$ as the starting material. This sample is termed HU-GO-3x.

Furthermore, the sorption activity of all graphene oxides towards aqueous zinc(II) and cadmium(II) ions was tested. $50 \mathrm{~mL}$ of each suspension with a concentration $5 \mathrm{mg} \mathrm{mL}^{-1}$ of graphene oxide was acidified using nitric acid $(0.5 \mathrm{~mL})$ and subsequently $100 \mathrm{~mL}$ of $5 \mathrm{wt} \%$ solution of cadmium (from cadmium nitrite) or zinc (from zinc nitrate) was added. The mixture was stirred for 24 hours and filtered. After multiple purification by distilled water the graphene oxide was dried and the concentration of sorbed zinc and cadmium was measured by ICP-OES. In addition, similar experiments were performed with solutions containing metals in lower concentrations (500 ppm). Finally, the third set of sorption experiments was performed using extremely diluted solutions (500 ppb of $\mathrm{Zn}^{2+}$ and $\mathrm{Cd}^{2+}$ ). In this experiment $25 \mathrm{mg}$ of graphene oxide was dispersed in $2500 \mathrm{~mL}$ of solutions containing $500 \mathrm{ppb}$ of zinc or cadmium (10 min ultrasonication; $300 \mathrm{~W}$ ). The mixture was stirred for 24 hours and separated by suction filtration. The concentrations of sorbed zinc and cadmium were measured by ICP-OES.

\section{Analytical techniques}

The morphology was investigated by scanning electron microscopy (SEM) with a FEG electron source (Tescan Lyra dual beam microscope). To conduct these measurements, the samples were placed on a carbon conductive tape. SEM and SEM-EDS measurements were carried out using a $10 \mathrm{kV}$ acceleration voltage.

For the measurement of Atomic Force Microscopy (AFM) images, the samples were dispersed in isopropanol $\left(1 \mathrm{mg} \mathrm{mL}^{-1}\right)$ and ultrasonicated for 15 minutes $(75 \mathrm{~W})$. After sedimentation, the suspension of graphene was dropped on a freshly cleaved mica substrate. These measurements were carried out on an Ntegra Spectra from NT-MDT. The surface scans were performed in a tapping (semi-contact) mode. Cantilevers with a strain constant of $1.5 \mathrm{kN} \mathrm{m}^{-1}$ equipped with a standard silicon tip with curvature radius lower than $10 \mathrm{~nm}$ were used for all measurements.

Combustible elemental analysis (CHNS-O) was performed using a PE 2400 Series II CHNS/O Analyzer (Perkin Elmer, USA). The instrument was used in CHN operating mode (the most robust and interference-free mode) to convert the sample elements to simple gases $\left(\mathrm{CO}_{2}, \mathrm{H}_{2} \mathrm{O}\right.$ and $\left.\mathrm{N}_{2}\right)$. The PE 2400 analyzer automatically performed combustion, reduction, homogenization of product gases, separation and detection. An MX5 microbalance (Mettler Toledo) was used for precise weighing of the samples (1.5-2.5 $\mathrm{mg}$ per single sample analysis). Using this procedure, the accuracy of CHN determination is better than $0.30 \%$ abs. Internal calibration was performed using $N$-phenyl urea.

The concentration of carboxylic acids was measured by alkalimetric titration using $0.1 \mathrm{M} \mathrm{NaOH}$ solution. The suspension of graphene oxide (200 $\mathrm{mg}$ ) was ultrasonicated for 30 minutes before the analysis.

High resolution X-ray photoelectron spectroscopy (XPS) was performed using an ESCAProbeP spectrometer (Omicron Nanotechnology Ltd, Germany) with a monochromatic aluminium X-ray radiation source $(1486.7 \mathrm{eV})$. The samples were placed on a conductive carbon tape homogeneously covered with the sample.

All samples were analysed by X-ray powder diffraction (XRD). Data collection was done with a Bruker D8 Discoverer diffractometer in Bragg-Brentano parafocusing geometry and applying $\mathrm{CuK}_{\alpha}$ radiation. The diffraction patterns were collected between $5^{\circ}$ and $80^{\circ}$ of $2 \theta$.

Simultaneous thermal analysis (STA) was performed using Linseis PT 1600 to $900{ }^{\circ} \mathrm{C}$ in an alumina crucible. The heating rate was set to $10{ }^{\circ} \mathrm{C} \mathrm{min}{ }^{-1}$ and the measurement was carried out in a dynamic synthetic air atmosphere $\left(20\right.$ vol\% $\mathrm{O}_{2} / 80 \mathrm{vol} \%$ $\mathrm{N}_{2}$ ) with a flow rate of $50 \mathrm{~mL} \mathrm{~min}^{-1}$.

Raman spectroscopy was conducted on an inVia Raman microscope (Renishaw, England) with a CCD detector in backscattering geometry. A DPSS laser $(532 \mathrm{~nm}, 50 \mathrm{~mW})$ with a $100 \times$ magnification objective was used for the Raman measurements. The instrument was calibrated with a silicon reference to give a peak position at $520 \mathrm{~cm}^{-1}$ and a resolution of less than $1 \mathrm{~cm}^{-1}$.

Fourier transform infrared spectroscopy (FT-IR) measurements were performed on a NICOLET iS50 FT-IR spectrometer (Thermo Scientific, USA). The FT-IR measurement was performed in transmission mode using $\mathrm{KBr}$ pellets. In addition, the measurement of FT-IR spectra was performed by FT-IR photoacoustic spectroscopy using a MTEC 300 photoacoustic detector in the range $4000-400 \mathrm{~cm}^{-1}$ at a resolution of $2 \mathrm{~cm}^{-1}$.

The ICP-OES measurements were performed using a SPECTRO ARCOS optical emission spectrometer (SPECTRO Analytical Instruments, Kleve, Germany) with radial plasma observation. The SPECTRO ARCOS features a Paschen-Runge spectrometer mount; the wavelength range between 130 and $770 \mathrm{~nm}$ can be simultaneously analyzed. The system is equipped with an air-cooled ICP-generator based on a free-running 27.12 MHz system. For sample introduction a cyclonic spray chamber and a modified lichte nebulizer were used. The following ICP operating parameters were applied: generator power $1450 \mathrm{~W}$, coolant flow $13 \mathrm{~L} \mathrm{~min}^{-1}$, auxiliary flow $0.8 \mathrm{~L}$ $\min ^{-1}$, nebulizer flow $0.75 \mathrm{~L} \mathrm{~min}^{-1}$, sample aspiration rate $2 \mathrm{~mL}$ $\min ^{-1}$. For calibration, commercially available multi-element standard solutions (Analytika) were used. The concentrations of calibrated elements were 0, 0.2, 1.0, 5.0, 10.0 and $20.0 \mathrm{mg} \mathrm{L}^{-1}$, respectively. $2 \mathrm{mg} \mathrm{L}^{-1} Y$ was used as an internal standard. All measurements were performed in $4 \mathrm{wt} \% \mathrm{HNO}_{3}$ as a matrix. To prepare liquid samples for ICP-OES analysis, the solid samples were weighed (approx. $5 \mathrm{mg}$ ) on a microanalytical balance and combusted by the Schöniger method. After combustion the closed Erlenmeyer flask was treated in an ultrasonic bath for several minutes. After absorption of combustion products (at least $2 \mathrm{~h}$ ) $50 \mu \mathrm{L}$ of $1 \mathrm{mg} \mathrm{mL}{ }^{-1} Y$ standard solution was added. Afterwards the liquid mixture was transferred from the glass flask to a plastic bottle. The flask was rinsed carefully with demineralized water which was added to the plastic bottle. The 
concentration of $\mathrm{HNO}_{3}$ was adjusted to $4 \mathrm{wt} \%$. Then the demineralized water was added to the plastic bottle to achieve the final volume of $25 \mathrm{~mL}$ (weighed). After mixing the solution was filtered and introduced into the spectrometer system.

Electrochemical characterization was performed by cyclic voltammetry using an Interface 1000 potentiostat (Gamry, USA) with a three electrode set-up. The glassy carbon working electrode (GC), platinum auxiliary electrode (Pt) and $\mathrm{Ag} / \mathrm{AgCl}$ reference electrode were obtained from Gamry (USA). For the cyclic voltammetric measurements, graphene was dispersed in $\operatorname{DMF}\left(1 \mathrm{mg} \mathrm{mL}^{-1}\right)$ and $3 \mu \mathrm{L}$ was evaporated on the glassy carbon working electrode. All potentials stated in the following section were measured against the $\mathrm{Ag} / \mathrm{AgCl}$ reference electrode. To measure the inherent electrochemistry, a phosphate buffer solution ( $\mathrm{PBS}, 50 \mathrm{mM}, \mathrm{pH}=7.2$ ) was used as the supporting electrolyte.

The sorption capacity towards $\mathrm{CO}_{2}$ and $\mathrm{CH}_{4}$ was obtained using a Micrometrics ASAP 2050 (Micrometrics Instrument Corporation, USA). The adsorption isotherms were measured for $\mathrm{CO}_{2}$ and $\mathrm{CH}_{4}$ at $303 \mathrm{~K}( \pm 0.1 \mathrm{~K})$ up to 10 bar. Before measurement each sample was processed by following the degassing procedure. Initially the sample was degassed at a temperature of $378 \mathrm{~K}$ and pressure of 0.15 Pa for 6 hours by connecting to a degas port. After weighing, the sample was transferred to the measurement port where the second treatment under the same conditions was carried out for 4 hours, followed by cooling down to $303 \mathrm{~K}$ and stabilization at this temperature for 3 hours. The standard measurement procedure with equilibration time $10 \mathrm{~s}$ was used.

\section{Results and discussion}

We successfully prepared once, twice and three times oxidized graphene oxides. By the terms second and third oxidation steps (or doubly and triply oxidized sample) we mean that the graphene oxide prepared by the first oxidation of graphite is repeatedly subjected to the respective oxidation procedure (Hofmann, Hummers or other methods). The as-prepared graphene oxide samples are shown in Fig. 1. All ST-GO show a black colour even after the third oxidation due to a relatively low concentration of oxygen functionalities. Also the HU-GO samples have a dark brown colour. Significant differences can be seen in the case of HO-GO, which changed colour from a typical dark green to light brown after the second oxidation. This indicates an extremely high degree of oxidation.

The microstructure of graphene oxides was analysed using SEM (Fig. 2). No significant differences can be seen in the SEM images, the wrinkled structure is apparent in all samples. The layered structure is also visible in triply oxidized samples.

In the next step the doubly and triply oxidized graphene oxides were characterized by atomic force microscopy (AFM). Note that the one-step oxidized graphene oxides had huge particle sizes with a typical thickness exceeding ten nanometers. Images are shown in Fig. 3. Few layered graphene oxide was only found for samples HO-GO- $2 x$ and HU-GO- $2 x$ whose thickness is approximately $6 \mathrm{~nm}$. ST-GO- $2 x$ is still not highly oxidized, hence thick particles were detected. In the case of

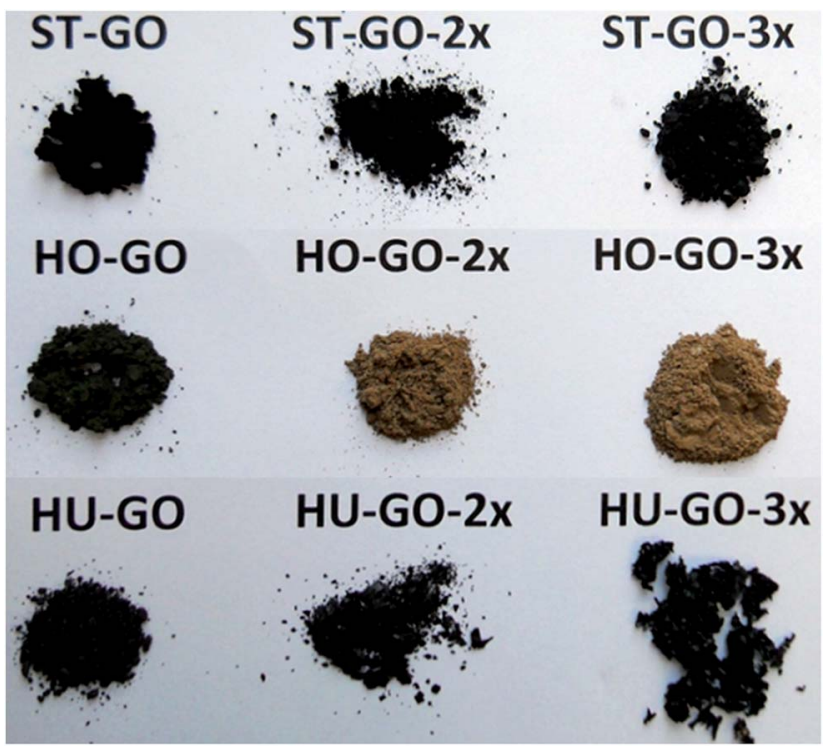

Fig. 1 The image of graphene oxide samples after multiple oxidation steps.

triply oxidized graphene oxides the thickness is lower compared to doubly oxidized graphene oxides. The decrease of thickness is most significant for ST-GO with multiple oxidations. This effect can also be assigned to the increase in interlayer distances which makes the exfoliation significantly easier by weakening of interlayer van der Waals bonds.

The chemical composition of graphene oxides was obtained by combustible elemental analysis (Table 1). The results confirmed that the second oxidation increased the oxygen content in GOs. A drastic increase in oxygen content was observed for ST-GO (from $25.2 \mathrm{wt} \%$ to $42.2 \mathrm{wt} \%$ ). On the other hand the second oxidation of HO-GO and HU-GO enhanced the oxygen concentration only slightly. The third oxidation also led to further increase in oxygen concentration, however the increase was lower compared to the second oxidation.

In addition we measured the concentration of carboxylic acids by acidimetric titration using $0.1 \mathrm{M}$ sodium hydroxide and a suspension of graphene oxide. The concentration of carboxylic acid functional groups such as $-\mathrm{COOH}$ in wt\% was $8.2 \mathrm{wt} \%, 12.2 \mathrm{wt} \%$ and $15.5 \mathrm{wt} \%$ for HU-GO, HU-GO- $2 x$ and HUGO-3x, respectively. In the case of ST-GO the concentration of carboxylic acids was $0.4 \mathrm{wt} \%, 3.8 \mathrm{wt} \%$ and $7.3 \mathrm{wt} \%$ for ST-GO, ST-GO-2 $x$ and ST-GO-3x, respectively. The concentration of carboxylic acids in HO-GO was $0.5 \mathrm{wt} \%, 0.9 \mathrm{wt} \%$ and $3.1 \mathrm{wt} \%$ for HO-GO, HO-GO- $2 x$ and HO-GO- $3 x$, respectively. A significant increase of carboxylic acid functional groups can be observed for all graphene oxides, however the oxidation route using permanganate is significantly more effective in increasing the concentration of carboxylic groups. A very high concentration of carboxylic acids is also documented by the results of other analyses (XPS, FT-IR) and led to a significant improvement of sorption capacity towards heavy metals.

To confirm these results, samples were analysed by high resolution XPS. The XPS survey spectra are presented in Fig. 4, 


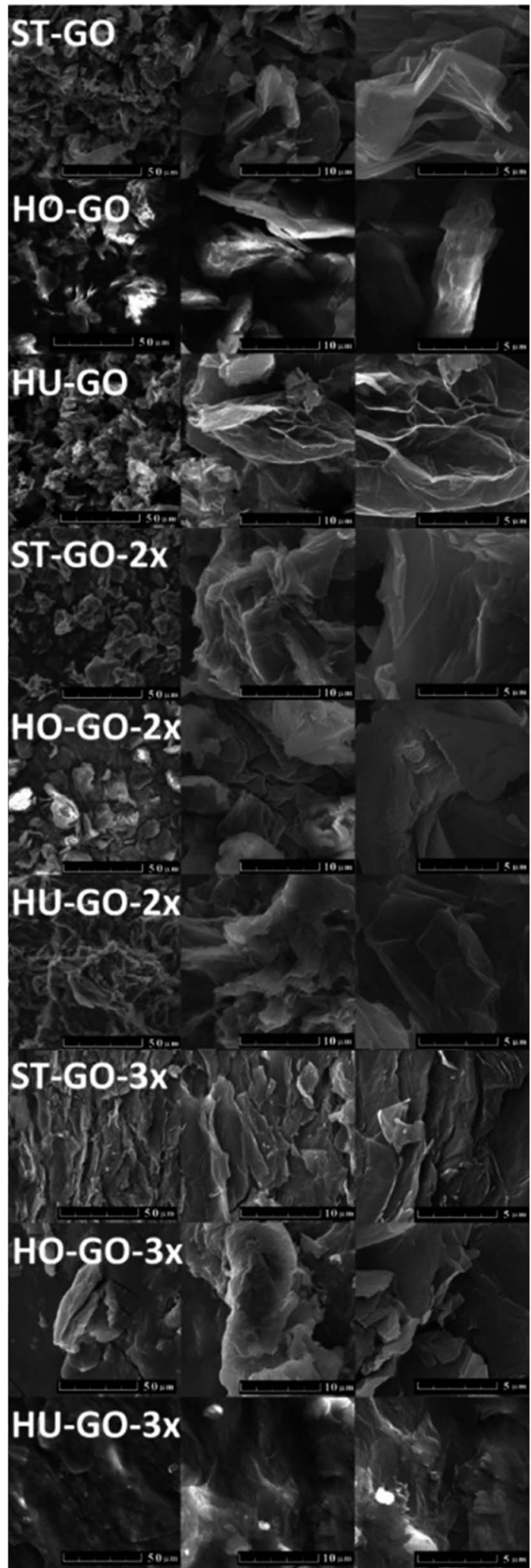

Fig. 2 SEM micrographs of graphene oxides.

where $\mathrm{C} 1 \mathrm{~s}$ and $\mathrm{O} 1 \mathrm{~s}$ peaks are clearly visible. The $\mathrm{C} 1 \mathrm{~s}$ peak was found at $284.45 \mathrm{eV}$ and the $\mathrm{O} 1 \mathrm{~s}$ peak at $533.0 \mathrm{eV}$. These spectra were used to calculate the concentrations of $\mathrm{C}$ and $\mathrm{O}$ (Table 2).

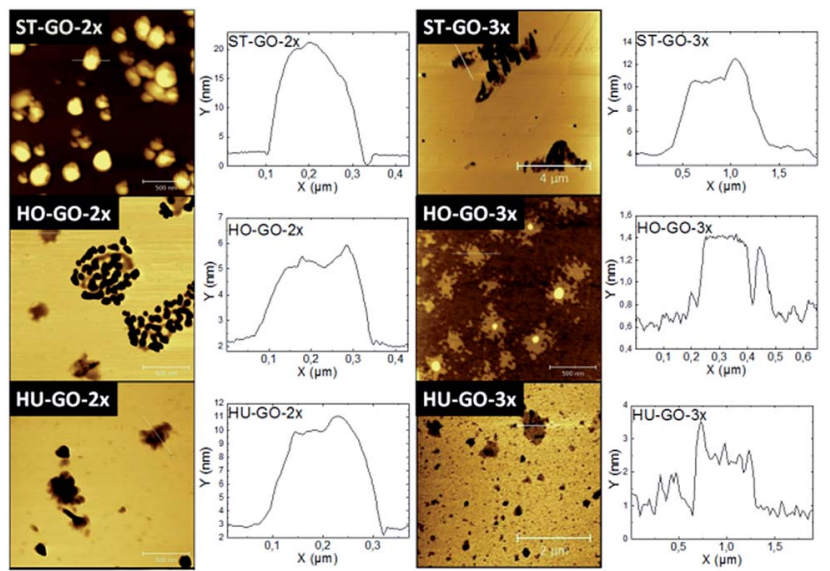

Fig. 3 AFM images of graphene oxides.

Table 1 Chemical composition of graphene oxides obtained by combustible elemental analysis in wt\%

\begin{tabular}{lllll}
\hline GO & $\mathrm{C}$ & $\mathrm{O}$ & $\mathrm{H}$ & $\mathrm{N}$ \\
\hline ST-GO & 73.70 & 25.20 & 1.10 & 0 \\
ST-GO-2x & 55.91 & 42.17 & 1.93 & 0 \\
ST-GO-3x & 52.08 & 45.77 & 2.07 & 0.08 \\
HO-GO & 54.02 & 43.68 & 2.27 & 0.03 \\
HO-GO-2x & 51.61 & 45.96 & 2.43 & 0 \\
HO-GO-3x & 51.00 & 46.54 & 2.35 & 0.11 \\
HU-GO & 56.97 & 40.94 & 2.02 & 0.07 \\
HU-GO-2x & 49.27 & 48.39 & 2.34 & 0 \\
HU-GO-3x & 40.43 & 56.46 & 3.01 & 0.1
\end{tabular}

The multiple oxidation led to a decrease of $\mathrm{C} / \mathrm{O}$ ratio. This is most clearly visible in the case of $\mathrm{HU}-\mathrm{GO}$, where a significant decrease of $\mathrm{C} / \mathrm{O}$ ratio can be seen even after the third oxidation procedure.

A detailed analysis of the $\mathrm{C} 1 \mathrm{~s}$ peak for all graphene oxides is shown in Fig. 5. High resolution C 1 s spectra were fitted to

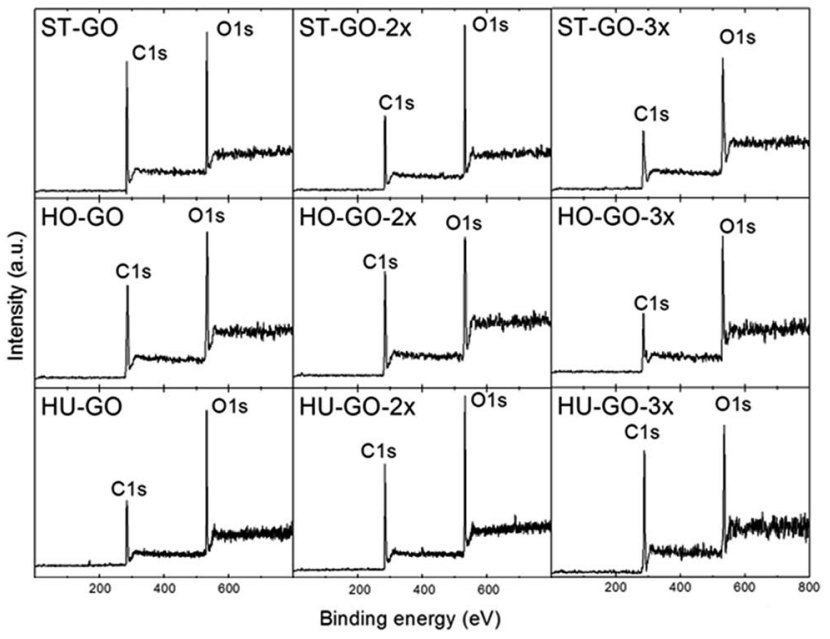

Fig. 4 XPS survey spectra of graphene oxides. 
Table 2 Chemical composition of graphene oxides obtained by high resolution XPS in at\%

\begin{tabular}{llll}
\hline GO & C & O & C/O \\
\hline ST-GO & 75.4 & 24.6 & 3.1 \\
ST-GO-2 $x$ & 69.1 & 30.9 & 2.2 \\
ST-GO-3x & 68.3 & 31.7 & 2.2 \\
HO-GO & 69.2 & 30.8 & 2.2 \\
HO-GO-2x & 66.1 & 33.9 & 1.9 \\
HO-GO-3x & 65.2 & 34.8 & 1.9 \\
HU-GO & 73.4 & 26.6 & 2.8 \\
HU-GO-2x & 68.5 & 31.5 & 2.2 \\
HU-GO-3x & 65.4 & 34.6 & 1.9 \\
\end{tabular}

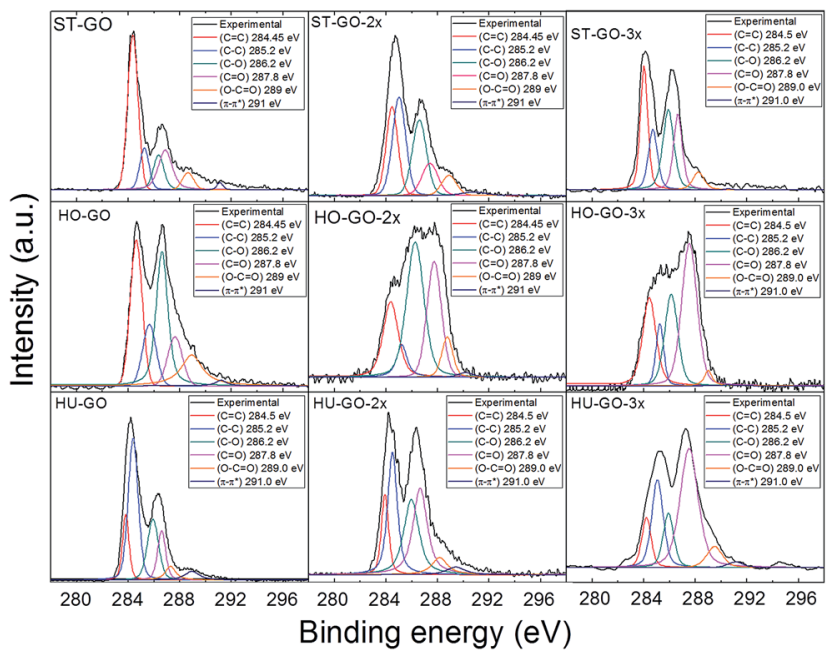

Fig. 5 Details of high resolution XPS spectra of the $C$ 1s peak, where fittings of the individual spectra show the possible carbon bonds.

quantitatively differentiate the six different carbon states: C-C $(284.5 \mathrm{eV}) ; \mathrm{C}=\mathrm{C}(285.2 \mathrm{eV}) ; \mathrm{C}-\mathrm{O}(286.2 \mathrm{eV}) ; \mathrm{C}=\mathrm{O}(287.8 \mathrm{eV}) ;$ $\mathrm{O}-\mathrm{C}=\mathrm{O}(289.0 \mathrm{eV}) ; \pi-\pi *$ interaction $(291 \mathrm{eV})$. The results are summarized in Table 3 documenting an increase of oxygen functionality concentrations for multiply oxidized samples and being in good agreement with the results of FT-IR spectroscopy as well as acidimetric titration. The multiple oxidation led to an increase of oxygen functionalities in the form of ketones and carboxylic groups. Indeed such a high concentration of oxygen functionalities implies notable changes in the electronic structure manifested by a change of colour as shown in the previous images.

XRD was used to investigate the structure of GOs (Fig. 6). The position of the (002) reflection shifted to lower values of $2 \theta$ according to the expected trend; a higher content of oxygen containing functional groups led to an increase of the interlayer distance (see Table 4). The shortest interlayer distance was found for ST-GO, whereas the largest interlayer spacing was observed for HU-GO-3x. Note that ST-GO also contained the graphitic phase as can be seen from Fig. 6 . In the case of HUGO-3 $x$ the interlayer distance was as high as $8.4 \AA$. Such a high increase of interlayer distances led to the weakening of van der
Table 3 Quantitative comparison of individual carbon stages in C 1s obtained by high-resolution XPS

\begin{tabular}{lcrrrrl}
\hline GO & $\mathrm{C}=\mathrm{C}$ & \multicolumn{1}{c}{$\mathrm{C}-\mathrm{C}$} & $\mathrm{C}-\mathrm{O}$ & $\mathrm{C}=\mathrm{O}$ & $\mathrm{C}(\mathrm{O})-\mathrm{O}$ & $\pi-\pi^{*}$ \\
\hline ST-GO & 47.57 & 12.33 & 12.67 & 19.87 & 5.95 & 1.58 \\
ST-GO-2x & 25.72 & 27.25 & 23.98 & 16.38 & 6.52 & 0.15 \\
ST-GO-3x & 28.00 & 17.03 & 27.48 & 20.64 & 6.59 & 0.26 \\
HO-GO & 29.28 & 15.67 & 26.97 & 13.30 & 12.98 & 1.8 \\
HO-GO-2x & 20.04 & 6.91 & 38.69 & 25.61 & 7.38 & 1.37 \\
HO-GO-3x & 21.41 & 14.16 & 22.74 & 36.72 & 4.12 & 0.85 \\
HU-GO & 13.52 & 42.58 & 20.83 & 12.62 & 5.63 & 4.82 \\
HU-GO-2x & 12.03 & 25.44 & 25.07 & 27.72 & 6.45 & 3.29 \\
HU-GO-3x & 10.13 & 21.75 & 12.57 & 46.13 & 7.34 & 2.08 \\
& & & & & &
\end{tabular}

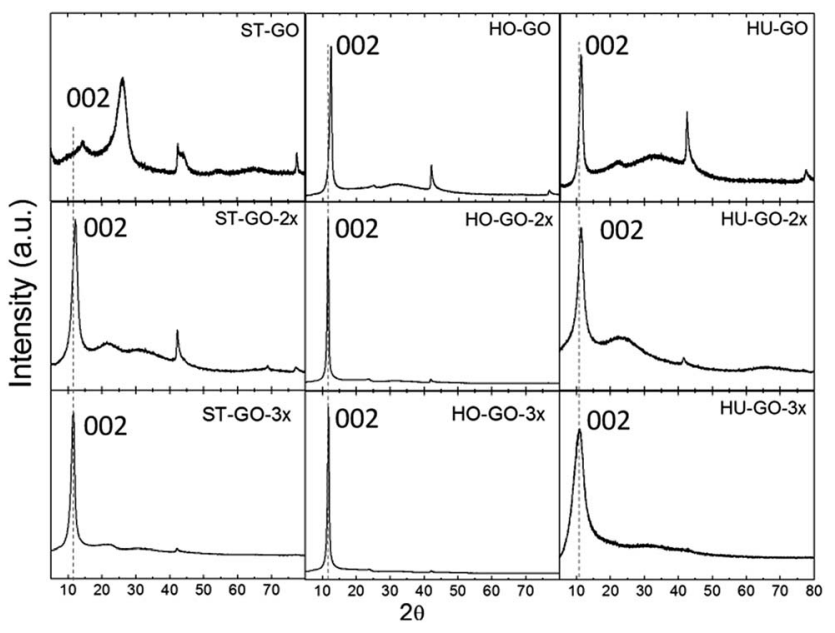

Fig. 6 XRD patterns of graphene oxides.

Waals forces between the individual layers and a significantly higher amount of monolayers can be seen by AFM, which was discussed in previous paragraphs. Apart from the (002) reflection, additional diffraction lines originating from (100), (101) and (110) planes can be observed at $42.3^{\circ} 2 \theta, 44.5^{\circ} 2 \theta$ and $77.4^{\circ}$ $2 \theta$ respectively. The intensity of these in-plane reflections is significantly reduced by repeated oxidation due to the formation of defects and covalent bonding of oxygen functionalities to the carbon atoms forming the graphene skeleton.

Table 4 The interlayer distances in graphene oxides obtained from the (002) reflection

\begin{tabular}{ll} 
GO & Interlayer distance $[\AA]$ \\
\hline ST-GO & 6.772 \\
ST-GO-2x & 7.284 \\
ST-GO-3x & 7.738 \\
HO-GO & 7.143 \\
HO-GO-2x & 7.548 \\
HO-GO-3x & 7.555 \\
HU-GO $2 x$ & 7.302 \\
HU-GO- $2 x$ & 7.442 \\
HU-GO-3x & 8.351
\end{tabular}


To obtain more information about the structure of the synthesized graphene oxides, their Raman spectra were measured. Two major bands corresponding to the D-band (1350 $\left.\mathrm{cm}^{-1}\right)$ and $\mathrm{G}$-band $\left(1580 \mathrm{~cm}^{-1}\right)$ were found in all spectra. The presence of the D-band indicates defects in the graphene layer (presence of $\mathrm{sp}^{3}$ bonded carbon atoms), while the G-band is associated with the $\mathrm{sp}^{2}$ bonded carbon atoms in the graphene layer (see Fig. ESI $\dagger$ ). We also calculated $I_{\mathrm{D}} / I_{\mathrm{G}}$ ratios, which are shown in Table ESI1. $\uparrow$ Thanks to the high luminescence of HOGO- $2 x$ and HO-GO- $3 x$ we were not able to measure these samples even at extremely low laser power. In the case of ST-GO samples an increase of $I_{\mathrm{D}} / I_{\mathrm{G}}$ ratio with multiple oxidation was observed in accordance with the rising concentration of oxygen functionalities. In the case of HU-GO another effect was observed: compared to HU-GO- $2 x$ the $I_{\mathrm{D}} / I_{\mathrm{G}}$ ratio was lower in HU-GO-3x. This may be explained in terms of the removal of some oxygen functionalities like epoxides and the predominant formation of ketone and carboxylic acid functionalities on the edges of graphene oxide sheets. The obtained $I_{\mathrm{D}} / I_{\mathrm{G}}$ ratio in $\mathrm{HU}-$ GO-3x is also strongly influenced by the background luminescence of this highly oxidized material.

The FT-IR spectra give information about the individual oxygen functionalities present in graphene oxide. The results are shown in Fig. ESI2. $\dagger$ In general, the ST-GO sample exhibits only weak intensities of absorption bands due to the high absorption coefficient of the material. The spectra of HO-GO, HU-GO and their multiply oxidized counterparts show an intensive and broad absorption band of $\mathrm{O}-\mathrm{H}$ stretching originating from hydroxyl functional groups around $3400 \mathrm{~cm}^{-1} \cdot^{26}$ The stretching of $\mathrm{C}=\mathrm{C}$ bonds is clearly visible at $1630 \mathrm{~cm}^{-1}$ (ref. 27) and the intensity of this absorption band decreases with multiple oxidations, since more $\mathrm{sp}^{3}$ bonded carbon atoms are formed with increasing degree of oxidation. The formation of $\mathrm{sp}^{3}$ hybridized carbon atoms originates from the increasing number of oxygen functionalities covalently bonded to the graphene skeleton which is clearly visible especially for samples prepared by chlorate oxidation methods. The multiple oxidation reactions with permanganate led to the oxidative removal of some oxygen functionalities, formation of defects and reduction of particle size since the carboxylic acid functionalities can be present only on the edges and defect sites of graphene oxide sheets. On the other hand, the intensity of the $\mathrm{C}=$ $O$ vibration band at $1720 \mathrm{~cm}^{-1}$ increases after multiple oxidation. ${ }^{27}$ This is in good agreement with the acidimetric titrations identifying an increased content of carboxylic acid groups. This vibration band has the highest intensity in HU-GO, where the highest concentration of carboxylic acids was also detected. In the case of ST-GO, the $\mathrm{C}=\mathrm{O}$ vibration band is not visible until the third oxidation cycle. The vibration band of $\mathrm{C}-\mathrm{O}$ functionalities in hydroxyls is observed around $1420 \mathrm{~cm}^{-1}$ and around $1060 \mathrm{~cm}^{-1} .{ }^{26}$ In addition, vibration bands at $970 \mathrm{~cm}^{-1}$ and 820 $\mathrm{cm}^{-1}$ can be observed in HO-GO samples. These bands may originate from the epoxide group. ${ }^{26}$

The thermal stability of graphene oxides towards oxidation was tested in a dynamic air atmosphere (Fig. ESI3 $\dagger$ ). Generally, two major thermal effects were observed on the DTA signal. The first effect at $\sim 200{ }^{\circ} \mathrm{C}$ corresponds to the exfoliation of graphene oxide. This thermal affect is accompanied by weight loss which is caused by the evolution of water, carbon dioxide and other products. ${ }^{28,29}$ In general, a higher weight change is observed for samples subjected to multiple oxidations since more oxygen functionalities are decomposed into volatile products like $\mathrm{CO}$ and $\mathrm{CO}_{2}$, where typically carboxylic acid functionalities decompose with $\mathrm{CO}_{2}$ evolution. The weight loss change also became sharper and faster for multiply oxidized samples. This may originate from a more uniform population of oxygen functionalities on the graphene oxide surface and particularly from the higher concentration of carboxylic acid functionalities. This is most pronounced for the three times oxidized graphene oxide with the highest concentration of carboxylic acid functionalities. The narrow step on the thermogravimetric curve and narrow sharp exothermic effect on the DTA signal indicate a rapid decomposition of oxygen functionalities like carboxylic acids in a very narrow temperature region. The second thermal effect at $\sim 600{ }^{\circ} \mathrm{C}$ is the oxidation (combustion) of the formed graphene.

Inherent electrochemistry was measured using cyclic voltammetry to obtain more information about the composition of the oxygen containing functional groups (Fig. 7). The peroxide group reduction potential is around $-0.7 \mathrm{~V}$, aldehyde around $-1.0 \mathrm{~V}$, epoxide around $-1.5 \mathrm{~V}$ and carboxyl around $-2.0 \mathrm{~V}$ (stated $v s . \mathrm{Ag} / \mathrm{AgCl}$ ). ${ }^{30}$ For $\mathrm{HO}-\mathrm{GO}$ the reduction started already

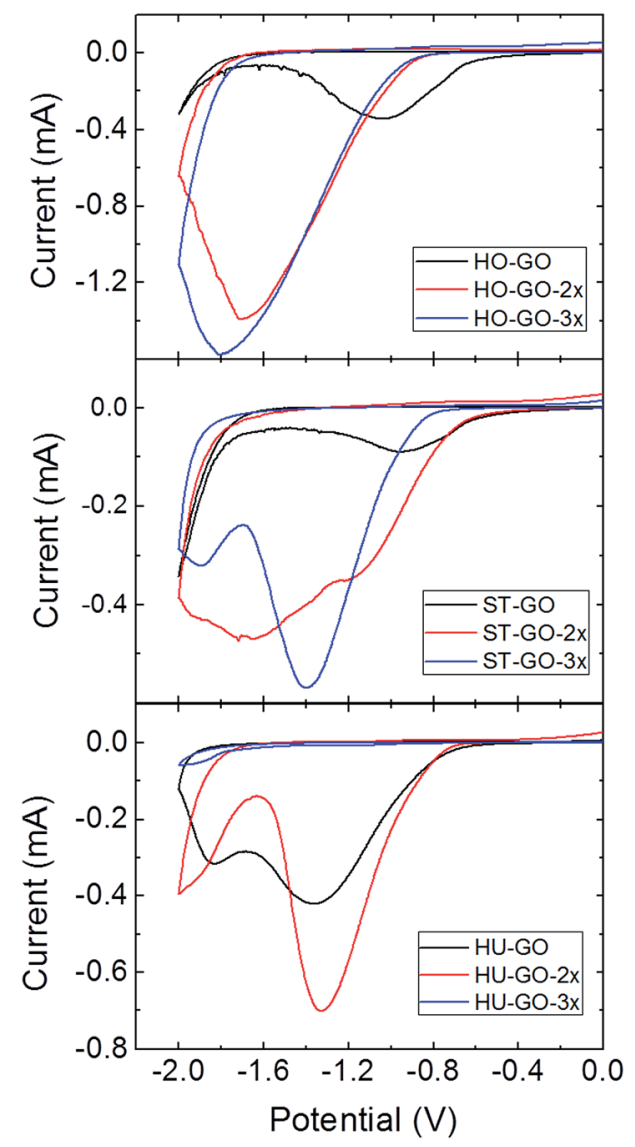

Fig. 7 Inherent electrochemistry of graphene oxides measured in PBS $\left(50 \mathrm{mM}, \mathrm{pH}=7.2\right.$, scan rate $100 \mathrm{mV} \mathrm{s}^{-1}$ ). 
at $-0.63 \mathrm{~V}$ and reached its maximum at $-1.05 \mathrm{~V}$. For doubly oxidized HO-GO- $2 x$ the reduction started at $-0.87 \mathrm{~V}$ and the maximum shifted to $-1.7 \mathrm{~V}$. HO-GO- $3 x$ showed very similar behavior to HO-GO-2x. This indicates the dominant presence of peroxide and epoxide groups in the HO-GO sample, while the multiple oxidations led to the formation of epoxides and also carboxylic acids. A similar trend was found for ST-GO and STGO- $2 x$. For ST-GO the reduction started at $-0.58 \mathrm{~V}$ and reached its maximum at $-0.96 \mathrm{~V}$, while for ST-GO- $2 x$ the reduction started at $-0.69 \mathrm{~V}$ and the maximum shifted to $-1.2 \mathrm{~V}$. The broad reduction signal in ST-GO- $2 x$ indicates the presence of several different functionalities like peroxides, epoxies and also traces of carboxylic acids. A slightly different behavior was observed for ST-GO- $3 x$. The reduction of ST-GO- $3 x$ started at $-0.87 \mathrm{~V}$ and the maximum shifted to $-1.4 \mathrm{~V}$ and the second maximum was close to $-1.8 \mathrm{~V}$. These results indicate the formation of carboxylic groups after the third oxidation, which is in good agreement with results of FT-IR as well as acidimetric titrations indicating the high concentration of carboxylic acids. For HU-GO and HU-GO- $2 x$ two reduction maxima were found. For HU-GO the reduction started at $-0.71 \mathrm{~V}$ and reached its first maximum at $-1.36 \mathrm{~V}$ and the second maximum at $-1.83 \mathrm{~V}$. This clearly shows the presence of epoxies as well as carboxylic acids. For doubly oxidized HU-GO- $2 x$ the reduction started at $-0.69 \mathrm{~V}$ and reached two maxima at $-1.33 \mathrm{~V}$ and $-1.92 \mathrm{~V}$. This is in good agreement with the results of FT-IR spectroscopy, where an increase of carboxylic acid concentration was observed. In the case of HU-GO- $3 x$ the reduction starts around $-1.60 \mathrm{~V}$ suggesting an oxidative removal of most electrochemically active oxygen functionalities while the remaining groups are carboxylic acids. In general, the shift of the reduction maxima to a lower potential indicates the formation of ketones and carboxylic acids as well as the decomposition of more labile groups such as epoxies by repeated oxidation procedures.

In order to demonstrate the applications of multiply oxidized graphene oxide we measured the sorption activity towards bivalent ions $\mathrm{Zn}^{2+}$ and $\mathrm{Cd}^{2+}$, since $\mathrm{Cd}^{2+}$ ions belong to highly toxic heavy metals. It has been described in the literature that different graphene oxides have various sorption capacities for different ions. This set of experiments can be useful for environmental issues such as removal and capture of toxic ions. The results of our experiments are summarized in Table 5 . The overall amounts of sorbed metals were measured by ICP-OES. It is obvious from this table that the amount of functional groups containing oxygen is the major factor for the sorption of ions from solutions. In general, oxygen functionalities like carboxylic acids can fix the metallic ions in the form of ionic compounds. Other oxygen functionalities can use the free orbitals for coordination of metallic ions, however this effect is weaker and the results indicate that mainly ketones can act as ligands for coordination of metallic ions.

This is documented by the differences in the sorption activity of graphene oxides prepared by chlorate and permanganate methods. In general, higher sorption capacity was observed for $\mathrm{Cd}^{2+}$ in comparison to $\mathrm{Zn}^{2+}$. The multiple oxidations also led to significant increase of sorption capacity, which is related to the increase of $\mathrm{C}=\mathrm{O}$ in ketones and carboxylic acids. This effect was observed most dominantly for HU-GO samples prepared by oxidation with permanganate, which is most apparent for triply oxidized HU-GO with a sorption capacity of $152.7 \mathrm{mg} \mathrm{g}^{-1}$ for $\mathrm{Cd}^{2+}$ and $65.6 \mathrm{mg} \mathrm{g}^{-1}$ for $\mathrm{Zn}^{2+}$. Sorption capacities reported in the literature were found to be lower than our values. The sorption capacities were $33.90 \mathrm{mg} \mathrm{g}^{-1}$ for $\mathrm{Cd}^{2+}, 23.26 \mathrm{mg} \mathrm{g}^{-1}$ for $\mathrm{Zn}^{2+}$ for biochar and $12.64 \mathrm{mg} \mathrm{g}^{-1}$ for $\mathrm{Cd}^{2+}$ and $12.90 \mathrm{mg} \mathrm{g}^{-1}$ for $\mathrm{Cd}^{2+}$ for active carbon. ${ }^{31}$ Powdered activated carbon impregnated alginate beads (ABPAC) as the adsorbent showed a sorption capacity of $56.0 \mathrm{mg} \mathrm{g}^{-1}$ for $\mathrm{Cd}^{2+} \cdot{ }^{32}$ Sunflower waste carbon calcium-alginate beads (SWC-CAB) showed the maximum sorption capacity of $23.6 \mathrm{mg} \mathrm{g}^{-1}$ for cadmium. ${ }^{33}$ Finally, chemically modified multiwalled carbon nanotubes showed a maximal sorption capacity of $32.68 \mathrm{mg} \mathrm{f}^{-1}$ for $\mathrm{Zn}^{2+} .^{34}$

Similar experiments were also performed with solutions containing 500 ppm of zinc or cadmium. The results are also summarized in Table 5 . High sorption capacity was also achieved for lower concentrations of metals, especially for the graphene oxide prepared by permanganate methods. Generally, the sorption capacity was only slightly lower compared to concentrated solutions. This suggests the high ability of multiply oxidized graphene oxide for applications focused on toxic ion removal from aqueous solutions. The last set of experiments with $500 \mathrm{ppb}$ solutions shows excellent sorption capabilities of multiply oxidized GO even at sub-ppm concentration of metallic ions. The increase of metallic ion

Table 5 Sorption capacity of graphene oxides in mg of metal per g of GO measured by ICP-OES. Two different solutions were used: 5 wt\%, 500 ppm and 0.5 ppm solution

\begin{tabular}{|c|c|c|c|c|c|c|}
\hline GO & $\mathrm{Zn}^{2+}, 5 \%$ & $\mathrm{Cd}^{2+}, 5 \%$ & $\mathrm{Zn}^{2+}, 500 \mathrm{ppm}$ & $\mathrm{Cd}^{2+}, 500 \mathrm{ppm}$ & $\mathrm{Zn}^{2+}, 500 \mathrm{ppb}$ & $\mathrm{Cd}^{2+}, 500 \mathrm{ppb}$ \\
\hline ST-GO & 9.1 & 3.7 & 1.7 & 3.5 & 1.6 & 0.1 \\
\hline ST-GO- $2 x$ & 10.8 & 11.5 & 7.9 & 12.3 & 4.0 & 1.1 \\
\hline ST-GO-3x & 11.9 & 12.6 & 8.1 & 13.2 & 5.6 & 1.1 \\
\hline HO-GO & 2.4 & 3.8 & 2.2 & 3.2 & 0.9 & 1.2 \\
\hline HO-GO-2x & 4.2 & 8.7 & 4.4 & 8.2 & 3.5 & 4.8 \\
\hline HO-GO-3x & 26.7 & 23.0 & 11.6 & 18.2 & 8.7 & 7.5 \\
\hline HU-GO & 10.4 & 21.5 & 10.7 & 19.2 & 7.4 & 7.6 \\
\hline HU-GO- $2 x$ & 32.2 & 82.7 & 32.1 & 63.6 & 21.4 & 12.2 \\
\hline HU-GO-3x & 65.6 & 152.7 & 44.7 & 67.5 & 21.9 & 24.1 \\
\hline
\end{tabular}



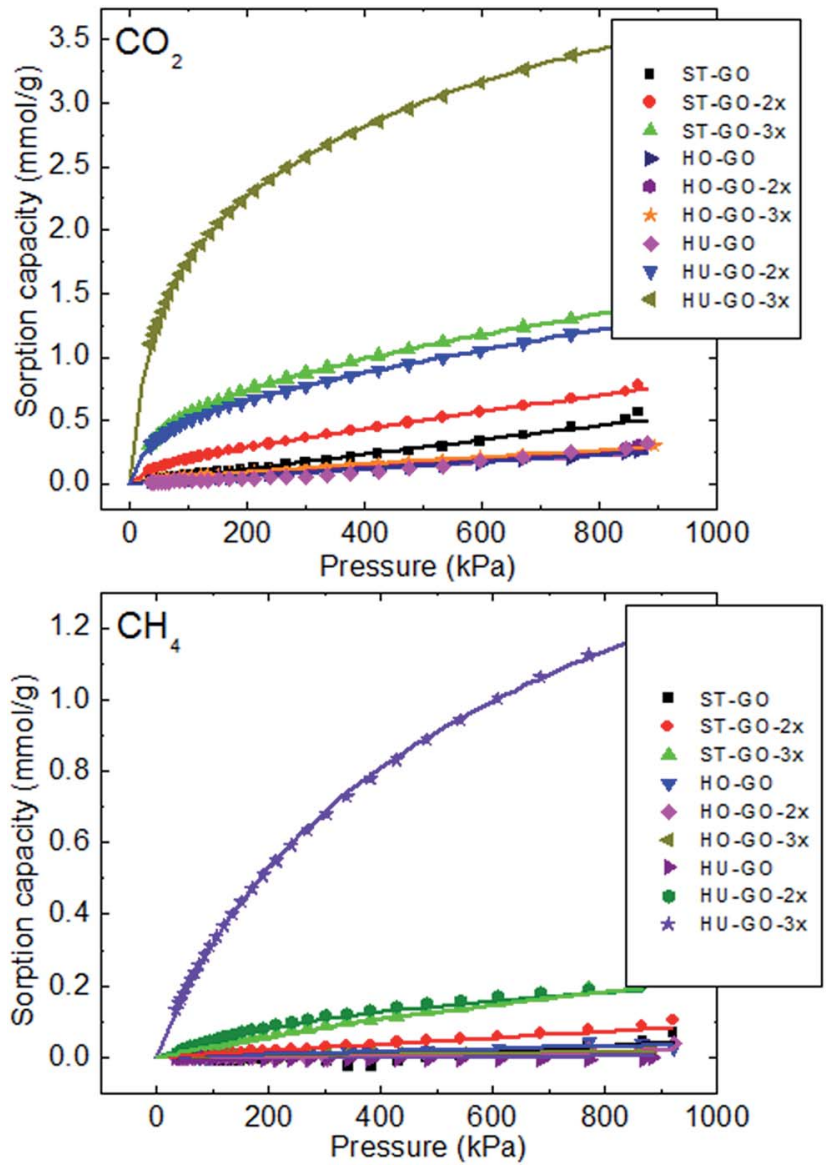

Fig. 8 Adsorption isotherms of $\mathrm{CO}_{2}$ and $\mathrm{CH}_{4}$ on graphene oxides. The measurement accuracy is about $0.03 \mathrm{mmol} \mathrm{g}^{-1}$.

concentration with repeated oxidation steps remains similar for high as well as low concentrations of metallic ions.

The sorption capacity towards carbon dioxide and methane for pressures up to $900 \mathrm{kPa}$ was investigated for graphene oxides (Fig. 8). Significant differences can be observed between the sorption capacity towards $\mathrm{CO}_{2}$ and $\mathrm{CH}_{4}$ indicating the possible application of graphene oxide for separation of $\mathrm{CO}_{2}$ from $\mathrm{CH}_{4}$ or the use of graphene oxide as a $\mathrm{CO}_{2}$ storage material. The sorption capacities for $\mathrm{CO}_{2}$ reaches the highest values for triply oxidized graphene oxide prepared by Hummers and Staudenmaier methods (3.54 $\mathrm{mmol} \mathrm{g}^{-1}$ and $1.43 \mathrm{mmol} \mathrm{g}^{-1}$, respectively). Similar trends were observed for $\mathrm{CH}_{4}$ adsorption capacity, however, the sorption capacity was about three times lower $\left(1.20 \mathrm{mmol} \mathrm{g}^{-1}\right.$ and $0.21 \mathrm{mmol} \mathrm{g}^{-1}$ for HU-GO-3x and ST-GO-3x, respectively). The shape of sorption isotherms could indicate the partial condensation of $\mathrm{CO}_{2}$ in the interlayer space at higher pressures. This also correlates with an increase of the sorption capacity with increasing interlayer distance as obtained by X-ray diffraction. The multiple oxidation also led to a decrease of individual sheet size which induced an increase of defect concentration and reduced sheet size, which means increase of edge concentration, where the adsorption dominantly takes place. The sorption mechanism will also be related to the chemical composition of graphene oxide surface. The sorption capacity correlates with the concentration of ketonic and carboxylic acid functional groups, which are dominantly present on the edges and defect sites of graphene oxide sheets. The shape of $\mathrm{CH}_{4}$ adsorption isotherm indicates the prevailing surface adsorption without any effect of condensation. The adsorption data for $\mathrm{CO}_{2}$ on graphene oxide can be well fitted by the dual-site Langmuir model using an equation:

$$
q=q_{1} \frac{b_{1} p}{1+b_{1} p}+q_{2} \frac{b_{2} p}{1+b_{2} p}
$$

where $q_{1}$ and $q_{2}$ are the saturated loadings for site A and site B, respectively, $b_{1}$ and $b_{2}$ stand for Langmuir parameters associated with the adsorption sites A and B, respectively, $p$ is the pressure and $q$ is the amount of adsorbed gas. The agreement with the dual-site Langmuir adsorption model is in line with the mechanism of $\mathrm{CO}_{2}$ adsorption on easily accessible surfaces, the edges and defects as adsorption sites A, and sites B identified as pore spaces formed by an increase of the interlayer distance (Fig. 8).

\section{Conclusions}

We have shown that the concentration and composition of oxygen functionalities in graphene oxides can be significantly tuned by multiple oxidations using both chlorate and permanganate routes. Multiple oxidations of graphene oxide led to an extremely high oxygen content and a significant increase of carboxylic acid content. This effect is most dominant in the case of triply oxidized graphene oxide obtained using permanganate, where the concentration of carboxylic acid exceeds $15 \mathrm{wt} \%$ and most of other functionalities were decomposed or oxidized to carboxylic acids. The high concentration of oxygen functionalities in graphene oxides, especially carboxylic acids can be used for the improvement of graphene oxide sorption capacity towards various metallic ions for environmental decontamination and pollution reduction applications. The optimisation and tuning of the composition and concentration of various oxygen functionalities is also a key parameter for the application of graphene oxide as a starting material for the synthesis of other graphene derivatives. In addition we investigated the possible applications of various graphene oxides for sorption of $\mathrm{CO}_{2}$ and $\mathrm{CH}_{4}$ which is an emergency application for the reduction of greenhouse gas pollution and also in industrial processes for natural gas purification, and hydrogen production by water gas shift reaction, where $\mathrm{CO}_{2}$ is the major byproduct. The significant increase of oxygen functionalities can be effectively used for the removal of various metal ions including heavy metals from water based solutions in environmental remediation applications. Multiply oxidized graphene oxide obtained by the Hummers method shows very high sorption capacity giving enormous application potential for heavy metal ion removal from an aqueous environment even at sub-ppm concentrations.

\section{Acknowledgements}

The project was supported by the Czech Science Foundation (GACR No. 16-05167S and 15-09001S) and by the financial support from the specific university research (MSMT No. 20SVV/2016). 


\section{Notes and references}

1 A. K. Geim and K. S. Novoselov, Nat. Mater., 2007, 6, 183-191. 2 J.-H. Chen, C. Jang, S. Xiao, M. Ishigami and M. S. Fuhrer, Nat. Nanotechnol., 2008, 3, 206-209.

3 Y. Fan, L. Wang, J. Li, J. Li, S. Sun, F. Chen, L. Chen and W. Jiang, Carbon, 2010, 48, 1743-1749.

4 K. S. Kim, Y. Zhao, H. Jang, S. Y. Lee, J. M. Kim, K. S. Kim, J. H. Ahn, P. Kim, J. Y. Choi and B. H. Hong, Nature, 2009, 457, 706-710.

5 M. Pumera, A. Ambrosi, A. Bonanni, E. L. K. Chng and H. L. Poh, TrAC, Trends Anal. Chem., 2010, 29, 954-965.

6 O. Jankovsky, P. Simek, D. Sedmidubsky, S. Huber, M. Pumera and Z. Sofer, RSC Adv., 2014, 4, 7418-7424.

7 O. Jankovsky, P. Simek, D. Sedmidubsky, S. Matejkova, Z. Janousek, F. Sembera, M. Pumera and Z. Sofer, RSC Adv., 2014, 4, 1378-1387.

8 P. Šimek, K. Klímová, D. Sedmidubský, O. Jankovský, M. Pumera and Z. Sofer, Nanoscale, 2015, 7, 261-270.

9 O. Jankovsky, P. Simek, K. Klimova, D. Sedmidubsky, S. Matejkova, M. Pumera and Z. Sofer, Nanoscale, 2014, 6, 6065-6074.

10 S. Gadipelli and Z. X. Guo, Prog. Mater. Sci., 2015, 69, 1-60.

11 Z.-X. Guo, J. W. Ding and X.-G. Gong, Phys. Rev. B: Solid State, 2012, 85, 235429.

12 H.-Y. Cao, Z.-X. Guo, H. Xiang and X.-G. Gong, Phys. Lett. A, 2012, 376, 525-528.

13 S. Bae, H. Kim, Y. Lee, X. Xu, J. S. Park, Y. Zheng, J. Balakrishnan, T. Lei, H. Ri Kim, Y. I. Song, Y. J. Kim, K. S. Kim, B. Özyilmaz, J. H. Ahn, B. H. Hong and S. Iijima, Nat. Nanotechnol., 2010, 5, 574-578.

14 Z. Sofer, P. Simek, O. Jankovsky, D. Sedmidubsky, P. Beran and M. Pumera, Nanoscale, 2014, 6, 13082-13089.

15 L. Staudenmaier, Ber. Dtsch. Chem. Ges., 1898, 31, 14811487.

16 W. Hummers and R. Offeman, J. Am. Chem. Soc., 1958, 80, 1339.
17 U. Hofmann and A. Frenzel, Kolloid-Z., 1934, 68, 149-151.

18 B. C. Brodie, Ann. Chim. Phys., 1860, 1860, 466-472.

19 U. Hofmann and A. Frenzel, Ber. Dtsch. Chem. Ges. A, 1930, 63, 1248-1262.

20 D. C. Marcano, D. V. Kosynkin, J. M. Berlin, A. Sinitskii, Z. Sun, A. Slesarev, L. B. Alemany, W. Lu and J. M. Tour, ACS Nano, 2010, 4, 4806-4814.

21 D. R. Dreyer, S. Park, C. W. Bielawski and R. S. Ruoff, Chem. Soc. Rev., 2010, 39, 228-240.

22 F. Li, X. Jiang, J. Zhao and S. Zhang, Nano Energy, 2015, 16, 488-515.

23 G. Z. Kyzas, E. A. Deliyanni and K. A. Matis, J. Chem. Technol. Biotechnol., 2014, 89, 196-205.

24 J. Wang, M. Liang, Y. Fang, T. Qiu, J. Zhang and L. Zhi, Adv. Mater., 2012, 24, 2874-2878.

25 V. Chabot, D. Higgins, A. Yu, X. Xiao, Z. Chen and J. Zhang, Energy Environ. Sci., 2014, 7, 1564-1596.

26 M. Mermoux, Y. Chabre and A. Rousseau, Carbon, 1991, 29, 469-474.

27 S. Stankovich, R. D. Piner, S. T. Nguyen and R. S. Ruoff, Carbon, 2006, 44, 3342-3347.

28 O. Jankovsky, S. Hrdlickova Kuckova, M. Pumera, P. Simek, D. Sedmidubsky and Z. Sofer, New J. Chem., 2014, 38, 5700-5705.

29 Z. Sofer, O. Jankovský, P. Šimek, D. Sedmidubský, J. Šturala, J. Kosina, R. Mikšová, A. Macková, M. Mikulics and M. Pumera, ACS Nano, 2015, 9, 5478-5485.

30 A. Y. S. Eng, A. Ambrosi, C. K. Chua, F. Šaněk, Z. Sofer and M. Pumera, Chem.-Eur. J., 2013, 19, 12673-12683.

31 D. Kołodyńska, J. Krukowska and P. Thomas, Chem. Eng. Sci., 2017, 307, 353-363.

32 A. Sigdel, W. Jung, B. Min, M. Lee, U. Choi, T. Timmes, S.-J. Kim, C.-U. Kang, R. Kumar and B.-H. Jeon, Catena, 2017, 148(1), 101-107.

33 M. Jain, V. K. Garg and K. Kadirvelu, Bioresour. Technol., 2013, 129, 242-248.

34 C. Lu and H. Chiu, Chem. Eng. Sci., 2008, 139, 462-468. 